\title{
A framework for automatically visualizing and recommending efficient routes
}

\author{
Stefan Fuest *, Monika Sester
}

Institute of Cartography and Geoinformatics, Leibniz University Hannover, stefan.fuest@ikg.uni-hannover.de, monika.sester@ikg.uni-hannover.de

* Corresponding author

\begin{abstract}
:
Due to an increasing traffic density in urban environments, the traffic management as well as the society needs to face various problems including congestion, air pollution or a higher probability of accidents. Therefore, it is getting more important to make road users aware of efficient route alternatives to obtain a better distribution of the traffic flow. Since the time for making route decisions is usually limited, the visualization of the information, which should be conveyed, needs to be prepared in a very clear and easily understandable format. In this approach, we propose a framework for automatically visualizing route efficiency based on various environmentally relevant scenarios. Our methods used to create the map visualizations are based on human perception of space, in order to communicate routes and traffic-related situations more intuitively. That is, humans are assumed to mentally abstract the geographic space using various types of distortions rather than perceiving the environment in its actual shape. Based on these concepts, we argue that a perceptionbased representation of the route, as well as the visual communication of temporary disturbances may not only simplify the navigation process, but also supports an awareness for the current traffic dynamics, which thus may influence route choice behavior towards a more altruistic behavior. In this paper, we further present two algorithmic approaches for automatically abstracting the geometry of a route in more detail, using cartographic generalization techniques - to present the road network in a way, how it might be perceived based on a certain traffic situation.
\end{abstract}

Keywords: Route visualization, cartographic generalization, route choice behavior, cognitive map, traffic management

\section{Introduction}

When navigating, the individual decision for a certain route depends on various factors and circumstances, including time, distance or route complexity, but also temporal factors like the current or expected traffic density (Papinski et al., 2009; Ben-Elia \& Avineri, 2015). Since the traffic volume in urban environments increases rapidly, in many cases the road network cannot cope with the growing number of vehicles any longer. As a consequence, road users and inhabitants need to face problems like traffic jam, air pollution or an increasing risk of accidents. We may argue that these problems are also caused by a non-optimal distribution of vehicles within the road network. To improve the overall quality of the traffic system, it is important for the traffic management to make road users aware of route alternatives - which are optimal from a societal point of view. However, the question is, how can we visualize the efficiency of a route for the overall traffic situation, so that the user of the traffic infrastructure will be convinced to decide for the recommended route, even though it might not be the most efficient route for the individual traveler? Current navigation systems propose different routes - visualized on metric maps, which show detail information. Research in cognitive psychology, however, suggests that people mentally abstract the geographic space when they communicate routes to others (e.g. when preparing a handdrawn sketch map), using distortions, simplifications and other generalization techniques, while focusing on the most relevant information necessary for navigation (Agrawala \& Stolte, 2001; Downs \& Stea, 1979). Maps are a powerful means to visually communicate traffic situations. Based on concepts from cognitive psychology, we argue that a clearer representation of the route, as well as the visual communication of temporal disturbances (which cause the selected route to be most optimal) may not only simplify the navigation process, but also supports an awareness for the current traffic dynamics, which thus may influence route choice behavior towards a more altruistic behavior.

In the scope of this paper, we present a framework for automatically visualizing route recommendations based on various environmentally relevant scenarios. We further focus on two algorithmic approaches, which intend to 
automatically visualize route efficiency using cartographic generalization and schematization techniques.

\section{Related Work}

\subsection{Route choice factors}

When using the traffic infrastructure, people usually tend to act in a selfish way, since they intend to travel from one place to another in an individually optimal way (Adoko et al., 2013; Ringhand \& Vollrath, 2018). Depending on the purpose of the trip, different factors like time, distance or the level of route complexity directly affect their route choices during everyday navigation. Thus, individual route choices usually aim to find a route, which might be efficient for an individual person, though not ideal from a societal point of view.

Route choice factors can be roughly divided into three major categories: 1) Individual differences, 2) road network-related factors and 3) temporary disturbances. The first category addresses individual differences; particularly factors that are highly dependent on the driver's personal requirements, behavior and preferences. The choice for a specific route usually depends on the purpose of the trip (Ramaekers et al., 2013). Further factors address the driver's patience, familiarity with the area, flexibility, time pressure or the driver's experience. Based on these individual route choices, the driver may have preferences for one or more factors that directly address the characteristics of the route. The majority of researchers (Papinski et al., 2009; Abdel-Aty et al., 1995) agrees that the time required to reach the target can be considered as the most relevant factor for choosing a route. In particular, drivers prefer travel time instead of distance measures for making route decisions. Another relevant factor that affects route choice behavior, is the route simplicity. Bailenson and collaborators (1998) argue that drivers prefer straight roads as compared to more complex, curvy shaped roads. Similarly, in case the area to be traversed consists of a very dense road network, drivers tend to avoid the most cluttered parts, in order to minimize the chance to accidentally choose a wrong turn. Importantly, route choice is not to be defined as a fixed decision that is only performed once, before starting the trip. Route choice can rather be classified into a pre-trip decision making process and spontaneous on-route decisions. On-route decisions are predominantly made in case there is a temporary factor affecting the efficiency of the pre-selected route. A very common case of temporarily influenced route choice behavior is adapting to on-route congestion (Papinski et al., 2009). In case of a trafficrelated event (e.g. congestion), which results in the traffic situation to deviate from the usual situation, all these factors affect, if the driver sticks to the previously planned route, or if he/she deviates from the original route choice.

\subsection{Cognitive aspects in route visualization}

Research in cognitive psychology suggests that people mentally abstract the geographic space when they communicate routes to others (e.g. when preparing a handdrawn sketch map), using distortions, simplifications and other generalization techniques, while focusing on the most relevant information necessary for navigation
(Agrawala \& Stolte, 2001; Downs \& Stea, 1973). Such a cognitive map can be basically described as a model, a simplified representation of a real-world geographic space (Tversky, 1993). In particular, when acquiring knowledge about a real world environment, people focus on essential information, while disregarding irrelevant information. That is, externalizations of people's cognitive maps (sketch maps) are an important means for communicating spatial knowledge about a specific area, which usually represent information in an abstract, schematized format (Lynch, 1960). Due to individually different experiences and knowledge about the environment, each person perceives and mentally structures the environment in different ways. However, when analyzing sketch maps regarding the elements included, researchers found that there are some recurring patterns, which are observable in most representations (Agrawala \& Stolte, 2001; Appleyard, 1970). Despite the large distortions of the real configuration of the world, sketched route maps usually preserve the topology of all relevant elements that are crucial for navigating along a route. In fact, a wellcomposed accurate route map does not need to be metrically correct, but rather communicate the correct qualitative information (Skubic et al., 2004) and the route's structure accurately enough for a traveler to arrive at the proper destination. Regarding elements included in sketch maps, Tversky \& Lee (1999) further report that instead of drawing the route using its original shape, people widely schematize elements like paths or intersections, which are commonly aligned to a right angle representation. Similarly, slightly curving paths are usually not drawn using their exact shape, but a simplified representation that approximates a straight line. Despite its geometric incorrectness, such a representation might still be an intuitive and efficient way of representing the environment, since, for route following, the road user does not need to be familiar with the exact shape of the road (Agrawala \& Stolte, 2001). Researchers further investigated that preserving distances of route segments does not seem to be important information for conveying routes (Tversky \& Lee, 1999). Here, it is rather assumed that, in a mental representation, longer distances are cognitively compressed relative to shorter distances depending on the information load they are associated with (Sadalla and Magel, 1980). Golledge and Zannaras (1973) further propose that there is a direct relationship between the cognized distance and the time required to traverse any given path. That is, the travel time based on the given traffic flow on a route is assumed to have an influence on the cognitive perception of the traveled distance (MacEachren, 1980; Saedi \& Khademi, 2019).

In a previous approach for efficiently communicating a specific route, Agrawala and Stolte (2001) developed a set of generalization techniques designed to improve the overall route map usability. Their idea was based on generalization techniques applied in hand-drawn maps, proposing that these types of maps clearly visualize the essential information which is relevant for navigation and omit irrelevant information. Thus, the information included in hand-drawn maps is assumed to be efficient for navigation purposes. Different from most of the previous approaches for visually communicating routes using 
cartographic generalization (Tufte et al., 1990; Sester, 2002), we aim to focus on the visualization of various routes within a road network, while recommending one specific route, which is most efficient due to a current traffic-related event. We present the road network in a way, how it might be perceived, while actually travelling along the route - using abstractions commonly applied in externalizations of human cognitive maps as an input. At the same time, we intend to make travelers aware of the consequences, a route choice might have on the overall traffic situation and on the individual itinerary. Additionally, we do not intend to prescribe a specific route to the user, but rather recommend a temporarily optimal route, while still providing the option to actively decide for alternative routes.

\section{The Framework}

To automate the visualization process, we propose a framework, which integrates different approaches of visually recommending efficient routes. In this context, various cartographic generalization techniques are applied, including feature selection, simplification, schematization and distortion of map objects; but also the use of symbolization and graphical variables. Figure 1 illustrates our proposed framework for automatically visualizing efficient routes based on different environmentally relevant scenarios.

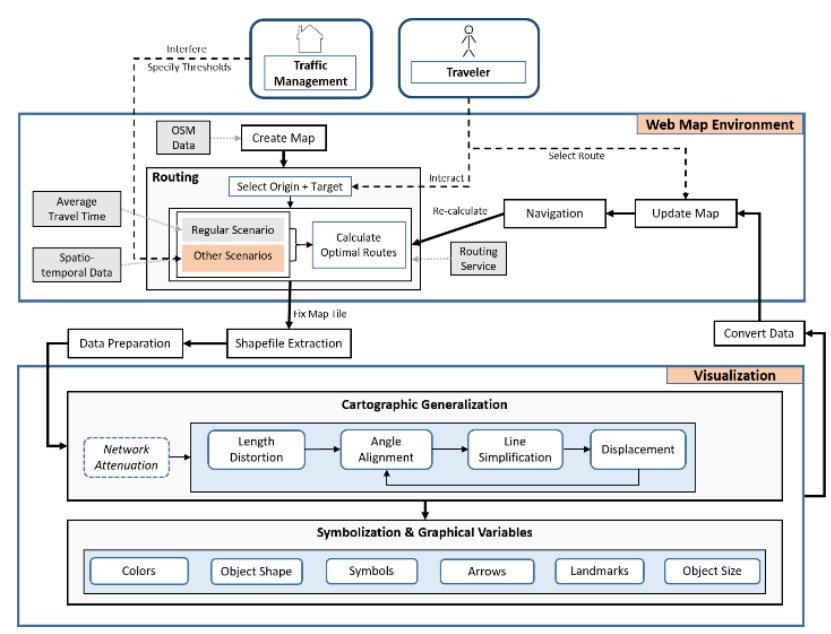

Figure 1. Framework for automatically visualizing environmentally efficient routes.

The framework involves two different types of agents: 1) The traffic management, which specifies regulations to improve the quality of the overall road traffic, and therefore directly interferes into the dynamics of the traffic infrastructure; and 2) the individual user of the traffic infrastructure (traveler), who intends to travel from one location to another in the most efficient way. The traffic management can interfere into the traffic dynamics, in case the - under usual circumstances - fastest route would be affected by a temporary disturbance, in order to improve the quality of the traffic infrastructure. The framework further integrates three different environmentally relevant scenarios (extendible to further scenarios), which each may require different approaches for efficiently visualizing route recommendations: 1) Avoiding congested roads (traffic density), 2) avoiding areas with high air pollution (e.g. particulate matter), and 3) avoiding locations with a high risk of accidents. These scenarios have been selected, since they represent some of the most important challenges, which the traffic management needs to face nowadays (May, 2013). Therefore, it is important to visually recommend routes, which are supportive for reducing the problems associated with these challenges.

\subsection{Web map environment}

The core of the framework consists of a web map environment, which also serves as an interface for the user to perform a routing from a start to a target location. The map environment is created using the JavaScript library Leaflet, while displaying Open Street Map data for the base map. For enabling the routing function, the application further integrates a routing engine (Leaflet Routing Machine), which provides route recommendations either for a regular routing scenario, or one of the three previously described environmentally relevant scenarios. In the regular routing scenario, the route suggestion is based on the fastest routes depending on the average travel time, as well as maximum two alternatives, calculated based on the same factor. In particular, this scenario will be selected by the system, in case the traffic management did not specify any further regulations. In some traffic situations, however, it can be necessary that the traffic management interferes into the traffic dynamics in order to improve the traffic system. For these scenarios, the traffic management can specify certain threshold values. Based on these thresholds, the routing service will calculate one most efficient route for each scenario. To perform the routing based on the different traffic-related scenarios, we use spatio-temporal data in different formats, which is associated to the vector data representing geographical features: 1) Current traffic density data for road segments in travel direction, 2) current particulate matter values for regions, and 3) recent accident data for the particular locations.

For the subsequent route visualization process, for the regular scenario, we focus our visualization on the three route options based on the average travel time. For the other scenarios (with additional regulations), we primarily visualize the calculated, currently best route option and additionally the three route options as calculated for the regular scenario. We additionally visualize temporarily inefficient routes, because we intend to convey both the efficiency of the recommended route, as well as the current inefficiency of other alternative routes. Despite their inefficiency for the traffic infrastructure, road users might frequently choose these routes, particularly in case it is a familiar route, the shortest or least complex route. Therefore, our approach is to make the traveler aware of the efficiency of route choices, as well as to communicate routes that should be avoided in order to act in a more altruistic way. Once the routes have been calculated, all geospatial data that is required for the subsequent visualization process is extracted from the map data. The data covers an area that is necessary to display all the suggested route alternatives. Since we intend to visualize efficient routes, we are primarily interested in vector data showing the routes and the adjacent road network, as well as additional features like object information that could be relevant to be included in a visualization. 


\subsection{Visualization}

The subsequent route visualization procedure is structured into two major parts that are designed as complementary, but can also be applied individually: First, the cartographic generalization, and second, the use of symbolization and graphical variables. Both types of visualizations include various different operators, based on cognitive psychology research. Importantly, some of the operators are not applied for all scenarios, whereas others will use a different method depending on the data involved. Similarly, some operators are mandatory for the visualization process, whereas others are optional. Same as for the scenarios itself, the framework can be flexibly extended by adding more visualization operators. Similar to other visualization techniques implemented as part of the framework using cartographic generalization, the distortion of the lengths of road segments integrates different approaches based on the particular scenario. In the regular scenario, road lengths of road segments are adjusted based on the perceived length of road segments. For the other traffic-related scenarios, we further address additional temporal factors like the current traffic density as an input for generalizing road segment length. Here, the idea is that people perceive road length depending on the actual travel time associated with traversing the route. (Saedi \& Khademi, 2019; Golledge \& Zannaras, 1973). A more detailed description of this approach is provided in section 4.1. of this paper.

In general, all visualization operators applied as part of the framework intend to visually optimize the most efficient route, while de-optimizing inefficient routes. For line simplification, for example, we use different approaches for either visualizing efficient or inefficient routes. For visualizing the recommended route, we apply the Douglas - Peucker Algorithm (Douglas \& Peucker, 1973) to the data, while using traffic-related data (e.g. traffic density) as an additional tolerance factor for deciding, if a point will be removed from the polyline. The smaller the traffic density, the more likely the point will be removed. In contrast, for visualizing inefficient routes, we developed a method for artificially distorting a line using the DouglasPeucker Algorithm in its reversed form (Vinatier \& Chauvet, 2017). The detailed method for this approach is described in section 4.2. of this paper.

Following the visualization process, the output vector data will be converted to the GeoJSON format for being represented in the web map environment. The initial map representation will then be replaced by our created visualization of the area including the routes. Ideally, the traveler now decides for the route, which has both been calculated and visually recommended as the most efficient route at a certain time and under certain circumstances.

\section{Algorithmic Approaches}

In the scope of this paper, we present two algorithmic approaches for automatically distorting the geometry of a route in more detail, using cartographic generalization techniques. For the described algorithms, we focus on the above presented scenario for avoiding congested roads.

\subsection{The length distortion algorithm}

Our motivation for developing an algorithm which automatically distorts the length of a given line segment is based on the assumption that travelers perceive road length depending on the actual travel time rather than the correct metrics of a road segment (Saedi \& Khademi, 2019; Golledge \& Zannaras, 1973, MacEachren, 1980). As described previously, travel time is further a relevant factor is models of spatial choice. Figure 2 below shows how the perceived distance between two locations along a route may deviate from the actual metric distance, depending on the current traffic density. In contrast to the metric distance between two points along a road segment, the perceived distance is not easily measurable in terms of metrics, since it is not a fixed value and varies depending on individual differences in human perception of space and time.
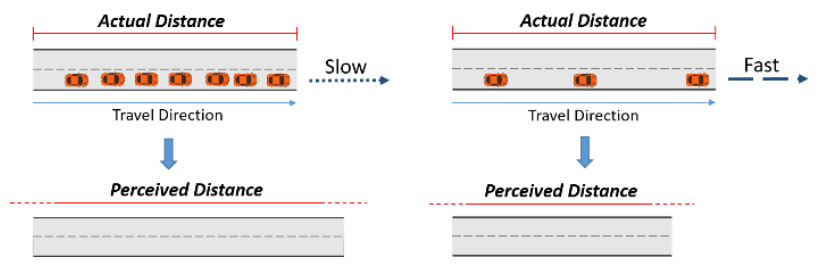

Figure 2. Actual length of a road segment as compared to the perceived length.

In order to distort the length of a line segment, we used the PUSH software (Sester, 2005), which has an enlarge functionality to scale objects by a factor. PUSH is a software program originally developed for automatically generalizing cartographic objects, particularly for removing spatial conflicts by means of displacement. It allows to describe the behavior of objects during the process of displacement very flexibly by defining various parameters, e.g. the allowed degree of deformation of an object or the minimum space required by an object. The parameters can be specified for each object individually. For automatically determining the enlarge factor, we first calculate an expected perceived length $p$ Len $(r)$ of a road segment using the following equation:

$$
p L e n(r)=\operatorname{len}(r) \cdot \frac{\emptyset \operatorname{dens}(r)+(\text { dens }(r)-\emptyset \operatorname{dens}(r)) \cdot w}{\emptyset \operatorname{dens}(r)}
$$

The calculations are based on the current traffic density dens $(r)$, measured for each individual road segment as compared to the average traffic density $\varnothing$ dens $(r)$ for the road segment during the same period of time. We further introduce a weight factor $w$, which allows to dynamically adjust the intensity of the deviation of the perceived length value from the original length value len $(r)$, using $w=$ $\{0,2\} \in \mathrm{Q}$

This weight factor provides an option to address individual differences regarding the perceived road length and provides the traffic management the option to interfere, in case there is a need to manually increase or decrease the intensity of distortions. We then calculate the enlarge factor as the ratio of the calculated perceived length of the line segment and its original length as follows:

$$
\text { enlarge }=\frac{p \operatorname{Len}(r)}{\operatorname{len}(r)}
$$


Once the line segments which have to be scaled are assigned the specific enlarge factors, the global optimization finds a holistic solution, taking all the other objects into account, i.e. displacing them appropriately, if needed. The resulting polyline will scale the line segments based on the input traffic density values. That is, high density segments are enlarged, whereas low density segments are reduced in length. In case the measured traffic density equals the average density for the road segment at a certain time, then the enlarge factor does not have any influence on the visualization of the segment. As the whole scene is treated in one optimization process, it is possible to also define larger values for the aura parameter for the corresponding line segments. This displacement of map objects can be very useful for visually establishing a clear distinction between different routes in a network.

\subsection{The line distortion algorithm}

Previous research suggests that the visualization of a road segment as a straight line may be associated with a very smooth movement in space (Thomson, 2004); and therefore also relates to a high traffic flow. In contrast, roads with a lower traffic flow (or higher traffic density) may be associated with a less smooth representation of a line, which deviates from a straight-line representation. Whereas for simplifying the complexity of a line, there already exist various commonly applied algorithms (e.g. the Douglas-Peucker algorithm), there are only few approaches, which intend to automatically distort a line (Vinatier \& Chauvet, 2017). In this section, we propose an algorithm, which artificially deforms the geometry of a given line segment using traffic-related data as an input for distortion.

The idea is that, in case of a temporarily high traffic density associated with a road segment, representing the line in a slightly more complex format may reflect the perceived viscosity of the traffic situation along the route segment. To do so, points are added on line segments and are slightly displaced from the straight-line connection in order to simulate an irregularity. The points are inserted randomly at either the left side of the original line, or the right side. Importantly, this algorithm only modifies line objects that are associated with a measured traffic density, which is larger than the average traffic density value for the segment during the same period of time and for the travel direction associated with the calculated routes. Thus, we define the original shape of the line as representing the overall average traffic density for each individual line segment. With an increasing traffic density, more points are inserted and the distance of the new points to the line increases.

Each line segment has exactly one starting point $P_{a}\left(x_{a}, y_{a}\right)$ and one end point $P_{b}\left(x_{b}, y_{b}\right)$-determined by the driving direction. In order to decide, how many additional points to create based on each existing line segment, and at which location they should be created, our proposed algorithm involves three different parameters, namely:

$n p:=$ Number of intermediate points created for each original line segment $f s:=$ Relative distance along a line segment from the starting point of a line segment $P_{a}\left(x_{a}, y_{a}\right)$ to base point $F$

$d:=$ Distance perpendicular to line segment from the base point $F$ to the new point $P_{n}\left(x_{n}, y_{n}\right)$

For determining the factors that define the number and the locations of the newly created points, we use traffic density data as an input. The number of intermediate points to be inserted for a line segment is calculated based on the intensity of traffic density associated with a road segment. For this, we categorize the intensity of traffic density and assign a $n p$ value to the input line segment as follows:

$$
\begin{gathered}
d r=\frac{\operatorname{dens}(r)}{\emptyset \operatorname{dens}(r)} \\
\forall a \in N \\
n p=\left\{\begin{array}{c}
0, \text { if } d r \leq 1.0 \\
a, \text { if } d r \in] 1.0+0.2 \cdot(a-1) ; 1.0+0.2 \cdot a]
\end{array}\right.
\end{gathered}
$$

Figure 3 illustrates, how the parameters $f s$ and $d$ are used for determining the location of two new points a certain distance away from the line segment. Point $P_{n 1}$ will be inserted on the left side of the line, whereas point $P_{n 2}$ will be inserted on the right side.

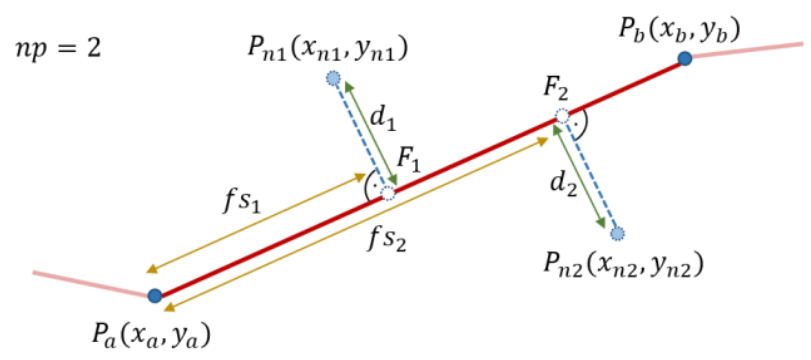

Figure 3. Parameters for calculating a new point location.

The parameter $f s$ describes the distance from the starting point $P_{a}\left(x_{a}, y_{a}\right)$ of the current line segment to a base point $F$ along the line segment, relative to the total distance between the points $P_{a}$ and $P_{b}$ (entire length of line segment). The relative length of $f s$ always depends on the number of points to be inserted. That is, because, for newly inserted, neighboring points, we intend to keep approximately equal distance intervals along the line segment. Therefore, we create normalized sections as a proportion of the total distance, with:

$$
d s=\frac{1}{n p+1}
$$

For each point $i$ in range $[1, n p+1]$ we then calculate

$$
f s=d s \cdot i
$$

Since we do not always want to distribute the newly inserted points using intervals of equal distance, we further add a randomization factor, which allows slight variations for the calculation of the length of parameter $f s$. Parameter $d$ specifies the distance to a new point $P_{n}$, perpendicular to the line segment $r$. Similar as for $n p$, the distance decision is also based on the ratio between the measured traffic density and the average traffic density. The larger the 
measured traffic density, the larger also the value for $d$, which means that for a road segment with a relatively high traffic density, a new point will be inserted a larger distance away from the original line segment. We define parameter $d$ as follows:

$$
d=w \cdot\left(\operatorname{dist} \cdot \frac{\operatorname{dens}(r)}{\emptyset \operatorname{dens}(r)}-\operatorname{dist}\right)
$$

The algorithm further allows to control the intensity of the distortions applied to the line by introducing an additional weight factor $w$, which can either increase or decrease the distance to the new point, and therefore also avoids distortions getting too extreme. Factor $w$ accepts values that are within a range $r$ with $w \in] 0,2]$. Once we have calculated the parameters $f s$ and $d$, we are able to calculate the point coordinates for determining the location of a new point $P_{n}\left(x_{n}, y_{n}\right)$. In a first step, we calculate the base point $F$ as follows:

$$
\begin{gathered}
d x=x_{b}-x_{a}, d y=y_{b}-y_{a} \\
F_{x}=x_{a}+f_{s} \cdot d x, F_{y}=y_{a}+f s \cdot d y
\end{gathered}
$$

Additionally, we randomize, if a new point will be inserted at the left side or the right side of the existing line segment. The direction perpendicular to the line segment at which the new point will be inserted can be defined by rotating the direction vector - either to the left or to the right while exchanging the gradients of $\mathrm{x}$ and $\mathrm{y}$. In case, the point should be inserted on the left side, we calculate:

$$
v x=-d y, v y=d x
$$

For inserting a point on the right side, we calculate:

$$
v x=d y, v y=-d x
$$

The new point coordinates are then determined as follows, with dist denoting the distance along the line segment:

$$
x_{n}=F_{x}+\frac{d \cdot v x}{d i s t}, \quad y_{n}=F_{y}+\frac{d \cdot v y}{d i s t}
$$

\section{Results \& Discussion}

\subsection{Length distortion}

We applied the road length distortion based on the enlarge functionality as implemented in the PUSH software to various route layouts in order to examine its performance and to evaluate how it handles geometric conflicts. As visualized in Figure 4, we first applied our approach to a single route, consisting of various line segments, which represent individual roads. Each line segment is associated with a traffic density value (vehicles per $\mathrm{km}$ ). For the enlarge parameter, we receive different factors for each line segment, based on the ratio between traffic density and average traffic density. Assuming an average traffic density of 40 for all line segments, for the relatively long lowermost road segment, for example, we calculate the value 0.6 as a factor, having 24 as an input value for the current traffic density. As a result, we receive a representation, with a correspondingly scaled the line length. When visualizing one single route, our program scales the individual road segments quite reliably depending on the input value for the enlarge parameter.

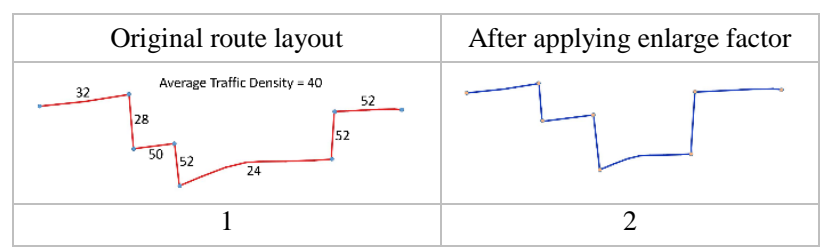

Figure 4. Effect of the enlarge factor on the distortion of line segments for a single route.

Since we are not only interested in applying this type of visualization to a single route, but also to multiple routes, which share the same starting- and end point, we particularly intend to achieve suitable road length distortion-based representations for a larger number of combined objects. The main problem in this case is that, when scaling each line segment based on an individual, defined factor, the locations of the starting and end points of the different routes may only match, in case additional distortions are applied to the line segments. Figure 5 below shows the original route layout including three different routes, route 1,2 and 3. (Map 1), as well as two resulting representations based on different definition of the parameters. Map 2 shows a visualization applying the enlarge parameter, using different enlarge factors for each line segment.

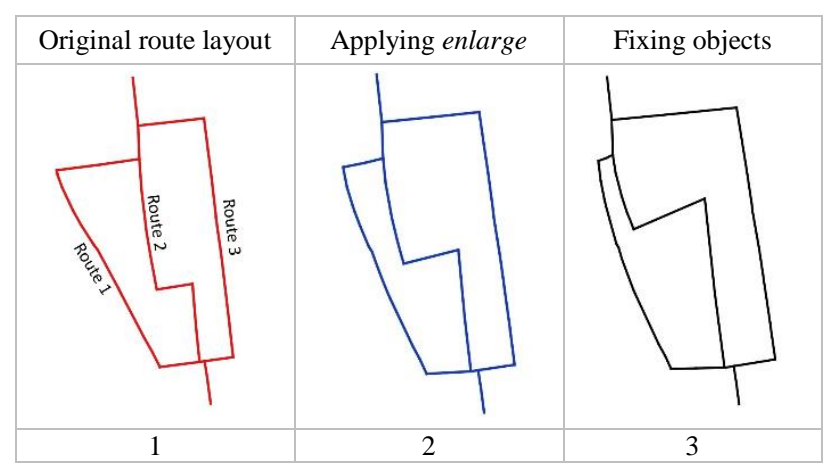

Figure 5. Effect of the enlarge factor and fixing objects on the distortion of line segments (multiple routes).

Route 2 is the shortest, whereas route 1 is the longest in terms of distance. In the representation of the original route layout, the route 1 appears to be not very efficient for traveling from a starting point to the target, as it looks much like a detour. In our scenario, however, the route on the left is associated with a relatively low traffic density as compared to the other two routes, and therefore would be recommended as the optimal route. Accordingly, the enlarge factors for route 1 are set relatively small, whereas for the other two routes, the factors are set to larger values. As a general observation, in map 2 now the left route is visualized as shorter as compared to the representation of the original layout, corresponding to the perceived road length based on the traffic situation. For the other two routes, we can observe the opposite effect, since here the route segments are generally represented as longer. Thus, the desired effect was achieved. However, if we consider the distortions of the individual line segments, we can observe that in most cases the differences in line length between the original and the distorted representation do not exactly match the scaling factor defined for enlarge. That is, because the system tries to satisfy all constraints in an optimal way, it is not possible to avoid additional distortions that occur due to mutual constraints regarding 
multiple objects, while still retaining the original topology of the road network. One possibility to control this behavior is to fix selected objects, so that they remain at their original location and are not affected by any distortions. In map 3, we fix the start and end point of the far left road segment. As a result, this line segment cannot be shifted, which results in other line segments being shifted and distorted even more distinctly. A side effect of this method is that the most efficient (Route 1) route now clearly appears to be the best route, since in this schematic representation it has the shortest length.

\subsection{Line distortion}

We tested the performance of the line distortion algorithm by varying the intensity of the distortion as well as the number of inserted points. Figure 6 shows four different sample line distortions generated based on our algorithm, illustrating how the shape of the resulting new line differs when varying the number of inserted points or the distance to the new point perpendicular to the line; while inserting new points randomly on either the left side or the right side of the line. In all cases, the dashed red line represents the original line segment, whereas the solid green line illustrates the new polyline. Figure A depicts the case that a relatively small number of additional points is inserted to form the new polyline, combined with a relatively small distance perpendicular to the original line segment. The resulting new line then only slightly deviates from the original line, equivalent to the associated traffic density value being only slightly higher than average. For figure $\mathrm{B}$, the same distance value is calculated, however more points are inserted. In that case the ratio between current and average traffic density is the same as for figure A, though the distance value $d$ has been decreased by the weight factor. Here, we perceive the resulting line as less smooth, since - given the larger amount of points inserted - the angles between two adjacent new line segments get smaller (if points are inserted on different sides of the original line); resulting into a more zigzag-like shape. When increasing $d$, this effect is even more noticeable, resulting into very large distortions, which hardly correspond to the shape of the original line any more (particularly visible in figure D). In order to avoid that distortions may get too extreme, we can reduce the distance between the points and the line by using a smaller weight value $w$ for the distance calculation $d$.

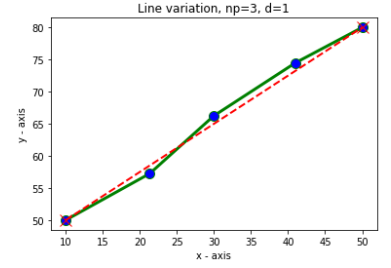

A

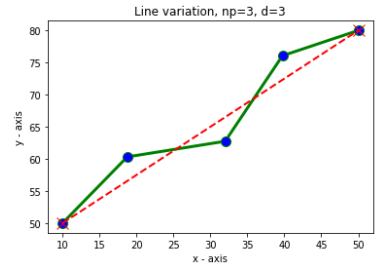

C

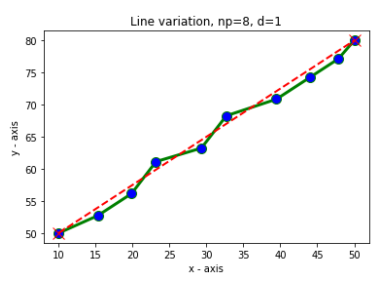

B

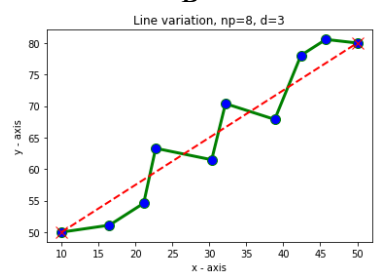

D
Figure 6. Line variations using different values for $n p$ and $d$.
Figure 7 illustrates the application of our algorithm to a polyline representing a route.

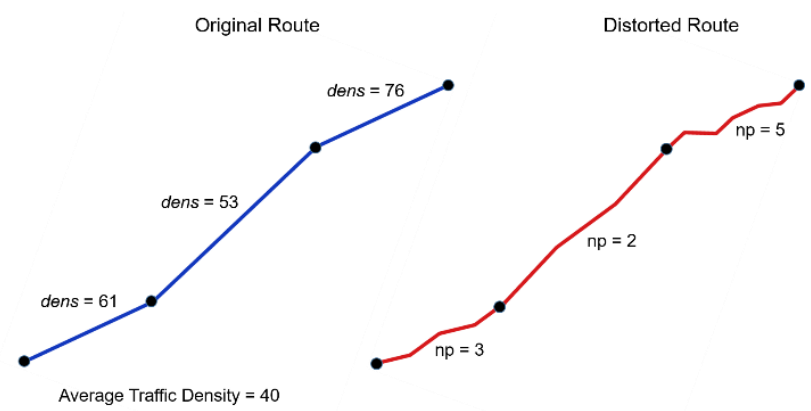

Figure 7. Different intensities of distortion for different line segments.

Since each route segment is associated with a different current traffic density value, our algorithm distorts each of the segments with a different intensity. For instance, the line segment located in the middle is only affected by slight distortions as compared to the original route representation, due to an only slightly increased traffic density. For the upper road segment, however, distortions are more distinct, as here, the current traffic density exceeds the average value more clearly. As a result, we obtain a representation of a line segment, which includes clearly recognizable distortions. This makes this route segment look more complex and may evoke the impression of a less smooth and less trouble-free movement in space when deciding for this particular road as compared to other options.

\section{Conclusion and Outlook}

In conclusion, our proposed framework intends to make travelers aware of the necessity to re-consider route choice behavior in order to improve the traffic dynamics and the overall traffic system, based on various environmentally relevant factors. Our method to approach this problem is to visually communicate the advantages of a recommended route, as well as disadvantages of inefficient routes to the road user, by using characteristics of cognitive processes associated to mapping a geographical space as an input. In particular, we intend to visualize the road infrastructure in a way that the traveler might perceive it while navigating, instead of presenting the accurate structural shape of the road network. We presented two algorithmic approaches to automatically visualize route recommendations by abstracting vector data of routes using cartographic generalization and schematization techniques. The resulting representation intends to make travelers aware of the consequences, temporarily efficient or inefficient routes may have on the individual journey and the entire traffic situation. In the end, our framework should support the improvement of the overall road traffic, by guiding travelers along a temporarily optimal route optimal in the sense of the traffic management.

Future work on this project will focus on the implementation of further algorithms to automate the process of visualizing route recommendations in various traffic situations. Subsequently conducted user studies for assessing the quality and suitability of the developed visualization methods focus on two major aspects: First, 
we will validate our visualizations by investigating the semantics, people will associate with the different types of visualizations, as well as the general acceptability and preferences for a visual representation. This also includes ways to inform the user about the distortions which have been applied. Based on this user study, we intend to obtain a representative collection of visualization methods that can serve as a guideline for visually recommending any type of route, under various circumstances. In a second step, we intend to investigate route choice behavior by comparing our developed visualizations with approaches commonly used by current routing services. Furthermore, we intend to extent the route visualization techniques to real-time navigation, by adapting the visualizations anytime the calculated optimal route differs from the previous calculation. Since, due to different factors influencing the traffic dynamics, the recommended route may change during the navigation process, adapting visualizations at different time steps during navigation is particularly relevant for improving road traffic in a sustainable way.

\section{Acknowledgement}

Funded by the Deutsche Forschungsgemeinschaft (DFG, German Research Foundation) - 227198829 / GRK1931.

\section{References}

Abdel-Aty, M. A., Kitamura, R., \& Jovanis, P. P. (1995). Investigating effect of travel time variability on route choice using repeated-measurement stated preference data (No. 1493).

Adoko, K. H., Pel, A. J., Hoogendoorn, R. G., \& van Arem, B. (2013). Modelling effects of social navigation on road traffic: The influence of penetration rates, altruism, and compliance. 8th TRISTAN, San Pedro de Atacama, Chile, 1-4.

Agrawala, M., \& Stolte, C. (2001, August). Rendering effective route maps: improving usability through generalization. In Proceedings of the 28th annual conference on Computer graphics and interactive techniques (pp. 241-249). ACM.

Appleyard, D. (1970). Styles and methods of structuring a city. Environment and behavior, 2(1), 100-117.

Bailenson, J. N., Shum, M. S., \& Uttal, D. H. (1998). Road climbing: Principles governing asymmetric route choices on maps. Journal of Environmental Psychology, 18(3), 251-264.

Ben-Elia, E., \& Avineri, E. (2015). Response to travel information: A behavioural review. Transport reviews, 35(3), 352-377.

Douglas, D. H., \& Peucker, T. K. (1973). Algorithms for the reduction of the number of points required to represent a digitized line or its caricature. Cartographica: the international journal for geographic information and geovisualization, 10(2), 112-122.

Downs, R. M., \& Stea, D. (1973). Cognitive maps and spatial behavior: Process and products (p. 25). na.

Golledge, R. G., \& Zannaras, G. (1973). Cognitive Approaches to the Analysis of Human Spatial Behavior. 1n Environmental Cognition, WH Ittelson, ed. New York: Seminar Press.
Lynch, K. (1960). The image of the city (Vol. 11). MIT press.

MacEachren, A. M. (1980) Travel time as the basis for cognitive distance. Professional Geographer 32:30-36.

May, A. D. (2013). Urban transport and sustainability: The key challenges. International journal of sustainable transportation, 7(3), 170-185.

Papinski, D., Scott, D. M., \& Doherty, S. T. (2009). Exploring the route choice decision-making process: A comparison of planned and observed routes obtained using person-based GPS. Transportation research part F: traffic psychology and behaviour, 12(4), 347-358.

Ramaekers, K., Reumers, S., Wets, G., \& Cools, M. (2013). Modelling route choice decisions of car travellers using combined GPS and diary data. Networks and Spatial Economics, 13(3), 351-372.

Ringhand, M., \& Vollrath, M. (2018). Make this detour and be unselfish! Influencing urban route choice by explaining traffic management. Transportation research part F: traffic psychology and behaviour, 53, 99-116.

Sadalla, E. K., \& Magel, S. G. (1980). The perception of traversed distance. Environment and Behavior, 12(1), 65-79.

Saedi, R., \& Khademi, N. (2019). Travel time cognition: Exploring the impacts of travel information provision strategies. Travel Behaviour and Society, 14, 92-106.

M. Sester (2005): Optimizing Approaches for Generalization and Data Abstraction, International Journal of Geographic Information Science, vol. $19 \mathrm{Nr}$. 8-9, pp. 871-897.

Sester, M. (2002). Application dependent generalizationthe case of pedestrian navigation. International Archives of Photogrammetry, Remote Sensing and Spatial Information Sciences, , 34(4), 291-296.

Skubic, M., Blisard, S., Bailey, C., Adams, J. A., \& Matsakis, P. (2004). Qualitative analysis of sketched route maps: translating a sketch into linguistic descriptions. IEEE Transactions on Systems, Man, and Cybernetics, Part B (Cybernetics), 34(2), 1275-1282.

Thomson, R. C. (2004). Bending the axial line: Smoothly continuous road centre-line segments as. In Proceedings 4th International Space Syntax Symposium, London, $U K$.

Tufte, E. R., Goeler, N. H., \& Benson, R. (1990). Envisioning information (Vol. 126). Cheshire, CT: Graphics press.

Tversky, B. (1993, September). Cognitive maps, cognitive collages, and spatial mental models. In European conference on spatial information theory (pp. 14-24). Springer, Berlin, Heidelberg.

Tversky, B., \& Lee, P. U. (1999, August). Pictorial and verbal tools for conveying routes. In International Conference on Spatial Information Theory (pp. 51-64). Springer, Berlin, Heidelberg.

Vinatier, F., \& Chauvet, M. (2017). A neutral model for the simulation of linear networks in territories. Ecological modelling, 363, 8-16. 